AJC-HEP-29

\title{
Low-Energy Interaction of a Cosmic String and an Extreme Dilatonic Black Hole
}

\author{
Kiyoshi Shiraishi* \\ Akita Junior College \\ Shimokitade-sakura, Akita-shi, Akita 010, Japan
}

(July 29, 2021)

\begin{abstract}
The interaction of a cosmic string and a maximally charged dilatonic black hole is studied in the low-velocity limit. In particular, the string-black hole scattering at a low velocity is investigated.

PACS number(s): 04.40.Nr, 04.70.Bw, 11.27.+d
\end{abstract}

Typeset using REVTEX

*e-mail: g00345@sinet.ad.jp, shiraish@air.akita-u.ac.jp 
Cosmic strings [1] are topological defects, which may have resulted from a certain phase transition in the early universe. When we take the infinitely thin limit of the string core, the cosmic string is characterized by a single dimensionless number $G \mu$, where $\mu$ is the mass density per unit length (in the unit $c=1$ ) and $G$ is the Newton constant. The spacetime outside of a straight cosmic string is described by the metric [2]

$$
d s^{2}=-d t^{2}+d r^{2}+\frac{r^{2}}{\nu^{2}} d \theta^{2}+d z^{2},
$$

where $\nu=(1-4 G \mu)^{-1}$.

If we use a new coordinate

$$
\vartheta=\frac{\theta}{\nu},
$$

the metric (11) reduces to the one of the flat spacetime

$$
d s^{2}=-d t^{2}+d r^{2}+r^{2} d \vartheta^{2}+d z^{2},
$$

but there is a deficit in the azimuthal angle since $\vartheta$ has the range

$$
0 \leq \vartheta<\frac{2 \pi}{\nu} .
$$

Then the space has a conical singularity along $z$-axis in the infinitely thin limit of the string. The deficit angle $\Delta$ is defined as [1.2]

$$
\Delta \equiv 2 \pi\left(1-\frac{1}{\nu}\right)=8 \pi G \mu .
$$

Linet [3] and Smith [4] have shown that a charged test particle placed near an infinite straight cosmic string feels a static self-force caused by the conical structure of the spacetime. It is also interesting to examine how the conical structure provides some kinematical effects in the motion of compact objects, since the interaction between cosmic strings and moving black holes may frequently occur in the early stage of the universe. In this paper, we construct the solitonic solution in Einstein-Maxwell-dilaton theory on the conical spacetime and investigate the motion of the solitonic object at a low velocity. 
The reason why we adopt this particular model is that the low-energy interaction between such dilatonic objects has been well known and we can utilize the result about it in the present case. The exact solution for an arbitrary number of charged dilatonic solitons has been found by several authors [5,6]. In the following, using the method of images, we obtain a solitonic solution on a conical space. We find that the solution reduces to the extreme dilatonic black hole solution on $R^{4}$ if the deficit angle vanishes.]

We consider a model described by the following action:

$$
S=\int d^{4} x \frac{\sqrt{-g}}{16 \pi G}\left[R-2(\nabla \phi)^{2}-e^{-2 \phi} F^{2}\right]+\text { (surface terms) }
$$

where $R$ is the scalar curvature, $\phi$ a dilaton, and $F_{\mu \nu}=\partial_{\mu} A_{\nu}-\partial_{\nu} A_{\mu}$ is the abelian gauge field strength.

We assume that the electric solution in the Einstein-Maxwell-dilaton theory takes the form [5,6]

$$
d s^{2}=-\frac{1}{V} d t^{2}+V d \boldsymbol{x}^{2}, A_{\mu} d x^{\mu}=\frac{1}{\sqrt{2}}\left(1-\frac{1}{V}\right) d t, e^{-2 \phi}=V
$$

Then $V$ satisfies

$$
\nabla^{2} V=0
$$

up to a number of delta functions in the right hand side of Eq. (8).

For example, one can find a solution:

$$
V=1+\sum_{\alpha} \frac{2 G m_{\alpha}}{\left|\boldsymbol{x}-\boldsymbol{x}_{\alpha}\right|}
$$

By studying the relation among the electric and dilatonic charge and mass of each solitonic object, we find that the solution describes the configuration that the $\alpha$-th nonrotating,

\footnotetext{
${ }^{1}$ The multi-soliton system in Einstein-Maxwell-dilaton theory on the spacetime with torus compactification has been studied by obtaining exact solutions and low energy interactions in a similar method [7].
} 
charged dilatonic "black hole" in the extreme limit with mass $m_{\alpha}$ located at $\boldsymbol{x}=\boldsymbol{x}_{\alpha}$ [5, 6]. Strictly speaking, there are singularities at $\boldsymbol{x}=\boldsymbol{x}_{\alpha}$. Nevertheless, we use the term "black hole" because the extreme case may still have generic properties of black holes in terms of their classical dynamics. Thus we use the term "black hole" without double quotation marks hereafter.

Now let us construct the black hole solution on a conical space.

When the string parameter $\nu$ is equal to an integer $p=1,2,3, \ldots$, we can derive the solution for $V$, which corresponds to the static configuration of one extreme dilatonic black hole and a cosmic string at the origin, by the method of images [⿴囗十 . The function $\Gamma_{p}\left(\boldsymbol{x}, \boldsymbol{x}^{\prime}\right)$ satisfying Eq. (8) and having periodicity $2 \pi / p$ in $\vartheta$ and $\vartheta^{\prime}$ can be expressed by the summation of $p$ images of $\Gamma\left(\boldsymbol{x}, \boldsymbol{x}^{\prime}\right)$, which is a solution of Eq. (8) in the Minkowski space:

$$
\Gamma_{p}\left(\boldsymbol{x}, \boldsymbol{x}^{\prime}\right)=\sum_{n=0}^{p-1} \Gamma\left(\boldsymbol{x}, \Lambda^{n} \boldsymbol{x}^{\prime}\right)=\sum_{n=0}^{p-1} \frac{1}{\left|\boldsymbol{x}-\Lambda^{n} \boldsymbol{x}^{\prime}\right|},
$$

where $\Lambda$ means the rotation by $2 \pi / p$ around the origin. Using the integral representation, we then obtain the solution for $V$ as follows:

$$
\begin{aligned}
\Gamma_{p}\left(\boldsymbol{x}, \boldsymbol{x}^{\prime}\right) & =\sum_{n=0}^{p-1} \frac{1}{\sqrt{r^{2}+r^{\prime 2}-2 r r^{\prime} \cos \left(\vartheta-\vartheta^{\prime}-2 \pi n / p\right)+\left(z-z^{\prime}\right)^{2}}} \\
& =\frac{1}{\pi \sqrt{2 r r^{\prime}}} \int_{u_{0}}^{\infty} \frac{d u}{\sqrt{\cosh u-\cosh u_{0}}} \sum_{n=0}^{p-1} \frac{\sinh u}{\cosh u-\cos \left(\vartheta-\vartheta^{\prime}-2 \pi n / p\right)} \\
& =\frac{1}{\pi \sqrt{2 r r^{\prime}}} \int_{u_{0}}^{\infty} \frac{d u}{\sqrt{\cosh u-\cosh u_{0}}} \frac{p \sinh p u}{\cosh p u-\cos p\left(\vartheta-\vartheta^{\prime}\right)}
\end{aligned}
$$

where

$$
\cosh u_{0}=\frac{r^{2}+r^{\prime 2}+\left(z-z^{\prime}\right)^{2}}{2 r r^{\prime}}
$$

We have the solution of $V$ which corresponds to asymptotically flat space:

$$
V=1+2 G M \Gamma_{p}\left(\boldsymbol{x}, \boldsymbol{x}^{\prime}\right)
$$

where $M$ is a constant. Note that $V$ is a solution of Eq. (8) even if $p$ is replaced by an arbitrary real number $\nu$. Consequently, we have the solution of $V$ corresponding to an extreme dilatonic black hole located at $\boldsymbol{x}^{\prime}=\left(r^{\prime}, \theta^{\prime}, z^{\prime}\right)$ in the conical space: 


$$
V=V_{C S}=1+\frac{2 G M}{\pi \sqrt{2 r r^{\prime}}} \int_{u_{0}}^{\infty} \frac{d u}{\sqrt{\cosh u-\cosh u_{0}}} \frac{\nu \sinh \nu u}{\cosh \nu u-\cos \left(\theta-\theta^{\prime}\right)} .
$$

This result can be extracted by using the mode expansion in terms of the Legendre functions [8]. $V_{C S}$ can be equivalently expressed as the sum of the Legendre functions:

$$
V_{C S}=1+\frac{2 G M \nu}{\pi \sqrt{r r^{\prime}}} \sum_{n=-\infty}^{\infty} Q_{\nu|n|-1 / 2}\left(\cosh u_{0}\right) e^{i n \theta}
$$

In the limit of $\nu \rightarrow 1, V_{C S}$ is reduced to $V$ in the flat space [5,6]. On the other hand, in the limit of $r^{\prime} \rightarrow 0, V_{C S}$ is reduced to

$$
V=1+\frac{2 G M \nu}{\sqrt{r^{2}+\left(z-z^{\prime}\right)^{2}}}
$$

which gives the metric representing a cosmic string trapped by a black hole. Thus the constant $M$ is identified to the black hole mass [9].

The cosmic string-extreme black hole solution in a general Einstein-Maxwell-dilaton model with an arbitrary dilaton coupling [6] can be constructed in the same manner.

Next we will present the low-energy interaction in the cosmic string-extreme black hole system.

The interaction energy of the maximally charged dilatonic black holes in $R^{4}$ at low velocities has been calculated without a long-distance approximation by making use of the exact, static solution (7) with (9) [10,11]. Since there are only two-body velocity-dependent forces in the multi-black hole system in this case, the general expression for the interaction energy of $O\left(v^{2}\right)$ of an arbitrary number of extreme dilatonic black holes can be easily obtained as: 10,12

$$
\begin{aligned}
H & =\sum_{\alpha} \frac{1}{2} m_{\alpha} \boldsymbol{v}_{\alpha}^{2}+\sum_{\alpha \beta} \frac{G m_{\alpha} m_{\beta}\left|\boldsymbol{v}_{\alpha}-\boldsymbol{v}_{\beta}\right|^{2}}{2\left|\boldsymbol{x}_{\alpha}-\boldsymbol{x}_{\beta}\right|} \\
& =\frac{1}{2} \mathcal{M} \boldsymbol{V}^{2}+\sum_{\alpha \beta} \frac{m_{\alpha} m_{\beta}\left|\boldsymbol{v}_{\alpha}-\boldsymbol{v}_{\beta}\right|^{2}}{4 \mathcal{M}}\left(1+\frac{2 G \mathcal{M}}{\left|\boldsymbol{x}_{\alpha}-\boldsymbol{x}_{\beta}\right|}\right),
\end{aligned}
$$

where $\boldsymbol{v}_{\alpha}$ is the velocity of the extreme dilatonic black hole with mass $m_{\alpha} . \mathcal{M}$ is the total mass, $\mathcal{M}=\sum_{\alpha} m_{\alpha} . \boldsymbol{V}$ is the velocity of the center of mass; $\boldsymbol{V} \equiv \sum_{\alpha} m_{\alpha} \boldsymbol{v}_{\alpha} / \mathcal{M}$. 
We use the method of images to obtain the interaction energy of extreme dilatonic black holes. We must note that the images of a certain black hole are located at $\Lambda^{n} \boldsymbol{x}$ and have the same mass $M$ and the velocity $\Lambda^{n} \boldsymbol{v}$. We also note that the sum of the interaction energy must be divided by the number of the images. The number $p$ is extended to a real number $\nu$ after completing the calculation.

Finally we get the following interaction energy up to $O\left(v^{2}\right)$ of the extreme dilatonic black hole with the mass $M$ and the velocity $\boldsymbol{v}$ on the conical space:[

$$
\begin{aligned}
H_{C S} & =\frac{1}{2} M v_{\|}^{2}+\frac{1}{2} M\left[1+\frac{2 G M}{\rho} \tan \frac{\pi(\nu-1)}{2 \nu}\right] \boldsymbol{v}_{\perp}^{2} \\
& =\frac{1}{2} M v_{\|}^{2}+\frac{1}{2} M\left[1+\frac{2 G M}{\rho} \tan \frac{\Delta}{4}\right] \boldsymbol{v}_{\perp}^{2},
\end{aligned}
$$

where $\rho$ is the distance between the cosmic string and the extreme black hole. $v_{\|}$is the $z$ component of the velocity of the extreme dilatonic black hole, while $\boldsymbol{v}_{\perp}$ is the component of the velocity perpendicular to the cosmic string. It is quite reasonable that the interaction energy diverges in the limit of $\Delta \rightarrow 2 \pi$.

The characteristic length scale of the interaction

$$
\rho_{0} \equiv G M \tan \frac{\Delta}{4} \approx 2 \pi G^{2} M \mu
$$

may be very small, if the string parameter $G \mu \simeq 10^{-6} \sim 10^{-5}$ as expected from cosmological considerations [1].

We shall study the classical scattering using the low-energy interaction energy. We consider the scattering of a maximally charged dilatonic black hole by a cosmic string by using the metric of moduli space [13].

Hereafter we assume that the black holes move in a plane which is perpendicular to the string. Therefore the moduli space of this configuration is reduced to be a two-dimensional

\footnotetext{
${ }^{2}$ For a case of a general dilaton coupling, since the interaction contains many-body, velocitydependent forces 10 12], the expression will be more complicated.
} 
space parameterized by the distance $\rho$, the azimuthal angle $\varphi$. For this two-body system, the metric on moduli space which spanned by the coordinate $\xi$ can be read from Eq. (18) as

$$
d s_{M S}^{2}=\gamma_{i j} d \xi^{i} d \xi^{j}=\gamma(\rho)\left(d \rho^{2}+\frac{\rho^{2}}{\nu^{2}} d \varphi^{2}\right)
$$

with

$$
\gamma(\rho)=1+\frac{2 G M}{\rho} \tan \frac{\Delta}{4}=1+\frac{2 \rho_{0}}{\rho} .
$$

Here $\varphi$ stands for the azimuthal angle which has a range $0 \leq \varphi<2 \pi$.

The path of the moving extreme black hole is determined by the geodesic equation on the moduli space [13], because the geodesic on the metric (20, 21) realizes the path of minimal energy. We find that the scattering trajectory satisfies the following differential equation:

$$
\left(\frac{d u}{d \varphi}\right)^{2}+\nu^{2} u^{2}=\frac{1}{b^{2}}\left(1+2 \rho_{0} u\right)
$$

where $u=1 / \rho$ and $b$ is the impact parameter.

The scattering angle $\Theta$ can be obtained by solving the equation (22) and expressed as:

$$
\begin{aligned}
\frac{\Theta}{\nu} & =\left(1-\frac{1}{\nu}\right) \pi+2 \arctan \left(\frac{\rho_{0} \nu}{b}\right) \\
& =\frac{\Delta}{2}+2 \arctan \left(\frac{\rho_{0} \nu}{b}\right) .
\end{aligned}
$$

The angle of deflection is written as the sum of the contributions from the deficit angle [1,2] and the Rutherford scattering [1]].

The quantum mechanical approach to the study of the scattering process is also possible by using the moduli space metric [14,11]. The Schrödinger equation on the moduli space, which is spanned by the coordinates $(\rho, \varphi, Z)$, can be read as 14, 11

$$
\begin{aligned}
i \hbar \frac{\partial}{\partial t} \Psi & =-\frac{\hbar^{2}}{2 M}\left(\frac{1}{\sqrt{\operatorname{det} \gamma_{i j}}} \partial_{k} \sqrt{\operatorname{det} \gamma_{i j}} \gamma^{k \ell} \partial_{\ell}+\frac{\partial^{2}}{\partial Z^{2}}\right) \Psi \\
& =-\frac{\hbar^{2}}{2 M}\left[\frac{1}{\gamma(\rho)}\left(\frac{1}{\rho} \frac{\partial}{\partial \rho} \rho \frac{\partial}{\partial \rho}+\frac{\nu^{2}}{\rho^{2}} \frac{\partial^{2}}{\partial \varphi^{2}}\right)+\frac{\partial^{2}}{\partial Z^{2}}\right] \Psi
\end{aligned}
$$

When we assume the wave function $\Psi$ can be decomposed as 


$$
\Psi=\psi(\rho, \varphi) e^{-i \frac{E}{\hbar} t} e^{i k Z}
$$

we get the following wave equation:

$$
\left[\frac{1}{\rho} \frac{\partial}{\partial \rho} \rho \frac{\partial}{\partial \rho}+\frac{\nu^{2}}{\rho^{2}} \frac{\partial^{2}}{\partial \varphi^{2}}+q^{2}\left(1+\frac{2 \rho_{0}}{\rho}\right)\right] \psi=0,
$$

where $q^{2} \equiv 2 M E / \hbar^{2}-k^{2}$. The equation (26) is the same as Eq. (4.2) in the paper on the Coulomb problem on a cone by Gibbons, Ruiz and Vachaspati [15], if we replace $\rho \rightarrow r, \varphi \rightarrow$ $\nu \phi, q \rightarrow k$ and $q^{2} \rho_{0} \rightarrow \mu K$. Thus we do not repeat the calculations on the scattering problem here. One can find that the differential cross-section diverges in the forward direction, but the divergence is shifted due to the presence of deficit angle of the spacetime [15]. This nature agrees with that of the classical scattering.

To summarize, we have studied the interaction of a cosmic string and maximally charged dilatonic black hole in the low velocity limit. The scattering of the black hole by a cosmic string has been investigated. The effect of the conical structure of the spacetime appears in the velocity-dependent force but may be very small for a "realistic" cosmic string.

We must note that our analyses are done in the low-velocity limit. The terms of higher order in $v$ and the radiational reaction may become important if the typical scales of the scattering process are very small. Moreover, cosmic strings may begin vibrating when the black holes approach, and then the nature of the interaction will be complicated. The study of these corrections needs additional approximation schemes, or requires numerical calculations. 


\section{REFERENCES}

[1] A. Vilenkin and E. P. S. Shellard, Cosmic Strings and other Topological Defects (Cambridge University Press, 1994).

[2] A. Vilenkin, Phys. Rev. D23, 852 (1981); Phys. Rep. 121, 263 (1985).

[3] B. Linet, Phys. Rev. D33, 1833 (1986).

[4] A. G. Smith, in: The Formation and Evolution of Cosmic Strings, G. W. Gibbons, S. W. Hawking and T. Vachaspati (eds.), Cambridge Univ, Press, 1990.

[5] G. W. Gibbons and K. Maeda, Nucl. Phys. B298, 741 (1988).

D. Garfinkle, G. Horowitz and A. Strominger, Phys. Rev. D43, 3140 (1991); (E) D45, 3888 (1992).

[6] K. Shiraishi, J. Math. Phys. 34, 1480 (1993).

[7] K. Shiraishi, "Extreme Dilatonic Black Holes on a Torus", preprint AJC-HEP-28, grqc/9511005.

[8] I. S. Gradshteyn and I. M. Ryzhik, Table of Integrals, Series, and Products (Academic Press, New York, 1965).

[9] M. Aryal, L. H. Ford and A. Vilenkin, Phys. Rev. D34, 2263 (1986).

D. V. Gal'tsov, Fortschr. Phys. 38, 945 (1990).

D. V. Gal'tsov and E. Masár, Class. Quantum. Grav. 6, 1313 (1989).

[10] K. Shiraishi, Nucl. Phys. B402, 399 (1993).

[11] K. Shiraishi, Int. J. Mod. Phys. D2, 59 (1993).

[12] K. Shiraishi, "Many-body systems in Einstein-Maxwell-Dilaton theory" preprint AJCHEP-26, gr-qc/9507029.

[13] N. Manton, Phys. Lett. B110, 54 (1982); ibid. B154, 397 (1985). 
R. S. Ward, Phys. Lett. B158, 424 (1985).

M. F. Atiyah and N. J. Hitchin, The Geometry and Dynamics of Magnetic Monopoles (Princeton University Press, Princeton, 1988).

[14] J. Traschen and R. Ferrell, Phys. Rev. D45, 2628 (1992).

[15] G. W. Gibbons, F. R. Ruiz and T. Vachaspati, Commun. Math. Phys. 127, 295 (1990). 